OPEN ACCESS

Edited by:

Xianglin Yuan,

Huazhong University of Science and

Technology, China

Reviewed by:

Alessandra Bettiol,

University of Florence, Italy

Nagaraja Nagre,

Eastern Virginia Medical School,

United States

*Correspondence:

Dan Chen

wzchendan@aliyun.com

Chengshui Chen

june8587@outlook.com

Specialty section:

This article was submitted to

Molecular Diagnostics and

Therapeutics,

a section of the journal

Frontiers in Molecular Biosciences

Received: 12 December 2020

Accepted: 26 April 2021

Published: 13 May 2021

Citation:

Zhu X, Lu S, Zhu L, Yu M, Wei T, ZhuX, Chen $D$ and Chen $C$ (2021) CXCR2

May Serve as a Useful Index of Disease

Activity in Interstitial Lung Disease

Associated With Primary

Sjögren's Syndrome.

Front. Mol. Biosci. 8:640779.

doi: 10.3389/fmolb.2021.640779

\section{CXCR2 May Serve as a Useful Index of Disease Activity in Interstitial Lung Disease Associated With Primary Sjögren's Syndrome}

\author{
Xiaofang Zhu ${ }^{1}$, Saisai Lu ${ }^{1}$, Lixia Zhu ${ }^{1}$, Mengjiao $Y u^{1}$, Tingting Wei ${ }^{1}$, Xiaochun Zhu ${ }^{1}$, \\ Dan Chen ${ }^{1 *}$ and Chengshui Chen ${ }^{2 *}$ \\ ${ }^{1}$ Department of Rheumatology, The First Affiliated Hospital of Wenzhou Medical University, Wenzhou, China, ${ }^{2}$ Department of \\ Pneumology, The First Affiliated Hospital of Wenzhou Medical University, Wenzhou, China
}

Background: Primary Sjögren's syndrome (pSS) is a chronic systemic autoimmune disease characterized by typical autoantibody production and lymphocytic-mediated exocrine gland damage. Interstitial lung disease (ILD) is a common complication of pSS and can be associated with a poor prognosis. However, the pathogenesis of ILD in pSS is still unclear.

Methods: In this study, we used RNA sequencing to investigate the gene-expression profile of the minor salivary glands (MSGs) from 36 patients with ILD-pSS and 128 patients with non-ILD-pSS.

Results: In the remarkably enriched chemokine-mediated signaling pathway, C-X-C motif chemokine receptor 2 (CXCR2), a receptor for interleukin-8, which participates in the activation of neutrophils, was found to be significantly elevated in both MSG and plasma from pSS patients with vs. without ILD $(p<0.001)$. Furthermore, the CXCR2 expression level in MSG and plasma was significantly associated with the diffusing capacity of the lungs for carbon monoxide, erythrocyte sedimentation rate, and EULAR Sjögren's Syndrome disease Activity Index in ILD-pSS.

Conclusion: Therefore, with its potential role in ILD progression in patients with pSS and its strong association with clinical manifestations of the disease, CXCR2 may serve as a useful index for disease activity in ILD associated with pSS.

Keywords: primary Sjögren's syndrome, interstitial lung disease, CXCR2, ESSDAI, DLCO

\section{INTRODUCTION}

Primary Sjögren's syndrome (pSS) is a systemic autoimmune disease characterized by the presence of organ-specific or non-specific autoantibodies and lymphocyte-mediated exocrine gland damage (Mavragani and Moutsopoulos, 2014). Although the major clinical manifestation of pSS is sicca symptoms, about $40 \%$ of patients develop a wide spectrum of extra-glandular involvement (Seror et al., 2014). Extra-glandular manifestations, especially interstitial lung disease (ILD), are associated with a poor prognosis and increased mortality in pSS (Roca et al., 2017; Sambataro et al., 2020). ILD has been observed in 9-75\% of patients with pSS (Kelly et al., 1991; Bellido-Casado et al., 2011; Nannini et al., 2013; Palm et al., 2013; Kreider and Highland, 2014; Jin et al., 2019; Natalini et al., 2019). In addition, ILD has been reported to have a cumulative incidence of $10 \%$ at 1 year after 
diagnosis of pSS, $20 \%$ after 5 years, and $47 \%$ after 15 years of disease (Nannini et al., 2013). Therefore, there is an urgent need to develop new diagnostic markers as well as potential therapeutic targets to improve the outcomes of patients with pSS with ILD.

Minor salivary gland (MSG) biopsy is a cornerstone in the diagnosis of pSS (Daniels and Whitcher, 1994; Caporali et al., 2008; Vissink and Bootsma, 2016; Chiu et al., 2020). MSG biopsies exhibiting focal lymphocytic sialadenitis, based on a focal score of one or more lymphocytic foci $\quad(>50$ lymphocytes $/ 4 \mathrm{~mm}^{2}$ ), are considered to be of great significance for pSS (Daniels and Whitcher, 1994; Caporali et al., 2008). Furthermore, a focus score of $\geq 4$ on an MSG biopsy is more frequently found in patients with ILD-pSS than in those with non-ILD-pSS (Kakugawa et al., 2018). Gene-expression profiling of MSGs has revealed a distinct gene-expression signature in patients with pSS compared with healthy control individuals (Hjelmervik et al., 2005). However, few studies have investigated differences in MSG gene expression between patients with ILD-pSS and those with non-ILD-pSS.

In this study, we investigated the clinical characteristics of and compared gene expression in MSGs from patients with ILD-pSS and non-ILD-pSS, with the aim of enhancing our understanding of the mechanisms underlying the progression of patients with pSS with ILD. In addition, we explored potential biomarkers for evaluating the disease severity of ILD-pSS in clinical practice.

\section{MATERIALS AND METHODS}

\section{Patients and Sample Preparation}

Patients with ILD-pSS $(n=36)$ and non-ILD-pSS $(n=128)$ were recruited from the First Affiliated Hospital of Wenzhou Medical University, China, between 1 January 2018 and 30 June 2020. All patients fulfilled the 2016 American College of Rheumatology (ACR)/EULAR classification criteria (Shiboski et al., 2017) or 2012 ACR classification criteria (Shiboski et al., 2012) for pSS and were evaluated for systemic involvement. A diagnosis of ILD was based on the findings of high-resolution computed tomography scans of the chest (Dong et al., 2018), combined with medical history and physical examination. All patients also underwent a MSG biopsy for diagnostic purposes.

Salivary glands and plasma samples were both collected at the time of MSG biopsies. All samples, clinical data, and EULAR Sjögren's Syndrome Disease Activity Index (ESSDAI) scores were obtained before the participants were given any systemic immunosuppressant or glucocorticoid to avoid confounding effects of these medications.

The study was approved by the ethics committee of the First Affiliated Hospital of Wenzhou Medical University (approval \#16024), and all patients gave their written informed consent.

\section{RNA Extraction, cDNA Library Preparation, and Sequencing}

Total RNA was isolated from frozen salivary gland samples using TRIzol Reagent (Invitrogen, Carlsbad, CA, United States) according to the manufacturer's instructions. RNA purity was
TABLE 1 | Clinical characteristics of patients with ILD-pSS and non-ILD-pSS.

\begin{tabular}{lccc}
\hline Characteristic & ILD-pSS & Non-ILD-pSS & $\boldsymbol{p}$ value \\
\hline$N$ & 36 & 128 & \\
Age, years, mean (range) & $60(43-86)$ & $49(28-79)$ & $0.000^{* *}$ \\
Male, $n$ (\%) & $7(19.4)$ & $18(14.1)$ & 0.645 \\
Anti-SSA antibodies, $n$ (\%) & $23(63.9)$ & $97(75.8)$ & 0.097 \\
Anti-SSB antibodies, $n$ (\%) & $7(19.5)$ & $44(34.3)$ & 0.144 \\
ESR (mm/h) & $32.41 \pm 18.52$ & $23.34 \pm 20.95$ & $0.020^{*}$ \\
CRP (mg/L) & $10.26 \pm 15.75$ & $4.93 \pm 9.30$ & 0.059 \\
IgG (g/L) & $17.52 \pm 4.44$ & $18.20 \pm 6.10$ & 0.544 \\
lgA (g/L) & $3.28 \pm 1.10$ & $3.40 \pm 1.43$ & 0.639 \\
IgM (g/L) & $1.63 \pm 1.30$ & $1.35 \pm 0.63$ & 0.097 \\
C3 (g/L) & $1.09 \pm 0.167$ & $1.05 \pm 0.22$ & 0.320 \\
C4 (g/L) & $0.21 \pm 0.17$ & $0.22 \pm 0.13$ & 0.509 \\
ESSDAl & $20.64 \pm 9.37$ & $7.02 \pm 7.85$ & $0.000^{* *}$
\end{tabular}

Data are means \pm standard error, except where otherwise indicated.

$\mathrm{p}<0.05,{ }^{* *} \mathrm{p}<0.001$ for ILD-pSS vs. non-ILD-pSS.

C3, complement 3; C4, complement 4; CRP, C-reactive protein; ESR, erythrocyte sedimentation rate; ESSDAI, EULAR Sjögren's Syndrome disease Activity Index; Ig, immunoglobulin.

checked using a Nano Photometer spectrophotometer (IMPLEN, Westlake Village, CA, United States). The quality of the RNA used to construct the cDNA library was verified (RNA integrity numbers >9) using an RNA 6000 Nano kit with the Bioanalyzer 2100 system (Agilent Technologies, Santa Clara, CA, United States). With input material of $3 \mathrm{mg}$ RNA per sample, we constructed sequencing libraries using the NEBNext Ultra RNA Library Prep Kit for Illumina (NEB, United States) and subsequently performed the sequence on an Illumina HiSeq platform. Then, using Cutadapt adapters and TrimGalore, raw reads were trimmed and low-quality reads were filtered. Qualitycontrol reports of sequence reads were obtained using FastQC software. Finally, the sequencing data were aligned to the human reference genome "hg38" using STAR software, and the read count files were filtered with low expression and normalized using the DESeq2 package.

\section{Enzyme-Linked Immunosorbent Assay}

Levels of C-X-C motif chemokine receptor 2 (CXCR2) and interleukin (IL)-8 in the plasma of patients with ILD-pSS and non-ILD-pSS were quantified using Human CXCR2 and Human IL-8 ELISA kits (Xitang Biology, Shanghai, China), respectively according to the manufacturer's instructions. The color was developed using 3,3',5,5'-tetramethylbenzidine (Sigma-Aldrich, St. Louis, MO, United States) and then measured using an ELISA plate reader (450 nm; Bio-Rad). All samples were measured in triplicate.

\section{Statistical Analysis}

The data analysis was conducted using $\mathrm{R}$ version 3.6.1. Twosample $t$-test was performed, calculating the mean \pm standard error (SE). Differentially expressed genes (DEGs) were identified according to the following criteria: adjusted $p$ value $<0.05$ and absolute value of $\log 2$ fold change $>1$. Pearson's correlation analysis was used to quantify the associations between geneexpression and clinical parameters, and Wilcoxon's test was applied to comparisons between subgroups. $p$ values $<0.05$ were considered significant. 


\section{Group $\doteqdot$ ILD-pSS $\underset{-1}{-}$ Non-ILD-pSS}
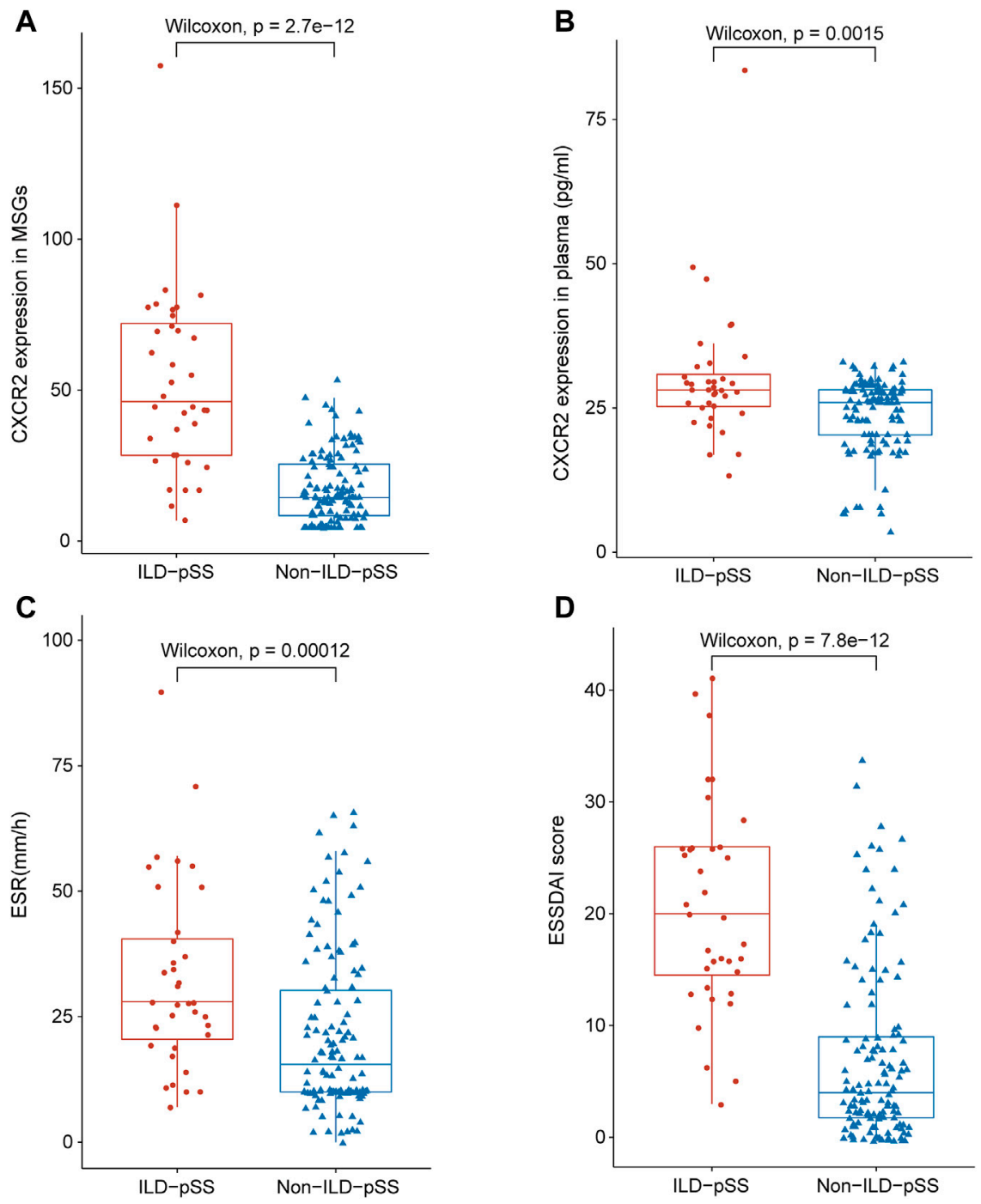

FIGURE 1 | Analysis of CXCR2 and IL-8 expression and clinical parameters in patients with ILD-pSS and non- ILD-pSS. CXCR2 gene expression by RNA sequencing (A), CXCR2 level in plasma (B), ESR (C), and ESSDAI scores (D) were significantly higher in patients with ILD-pSS vs. non-ILD-pSS patients. Horizontal lines, from bottom to top, denote the minimum, 25th percentile, median, 75th percentile, and maximum.

\section{RESULTS}

\section{Patient Characteristics}

The characteristics of the 36 patients with ILD-pSS and the 128 patients with non-ILD-pSS are detailed in Table 1. The mean \pm SE age of patients with ILD-pSS was $60 \pm 11.9$ years, which was significantly higher than that of patients with non-ILD-pSS ( $49 \pm 12.8$ years). Both groups had similar proportions of men (19.4 and $14.1 \%$, respectively) and higher proportions of women (80.6 and $85.9 \%$, respectively).

We also compared the differences in other clinical features between the two groups. Mean $( \pm$ SE) ESSDAI scores were elevated in those with ILD-pSS (20.64 \pm 9.37) and significantly higher than in patients with non-ILD-pSS (7.02 $\pm 7.85, p<0.001$; Figure 1D). Moreover, the mean $( \pm \mathrm{SE})$ erythrocyte sedimentation rate (ESR) was higher in patients with ILD-pSS $(32.41 \pm 18.52 \mathrm{~mm} / \mathrm{h})$ vs. non-ILDpSS $(23.34 \pm 20.95 \mathrm{~mm} / \mathrm{h}, p<0.001$; Figure 1C). Likewise, mean $( \pm$ SE) C-reactive protein levels were higher in ILD-pSS $(10.26 \pm 15.75 \mathrm{mg} / \mathrm{L})$ than in non-ILD-pSS $(4.93 \pm 9.30 \mathrm{mg} / \mathrm{L}$, $p=0.059$; Table 1), although this did not reach statistical significance. There were no significant differences between the two groups in the presence of anti-SSA antibodies $(p=0.097)$ or anti-SSB antibodies $(p=0.144)$, or in the levels of immunoglobulin (Ig)G, IgA, IgM, C3, or C4 $(p=0.544$, $0.639,0.097,0.320$, and 0.509 , respectively; Table $\mathbf{1}$ ). 


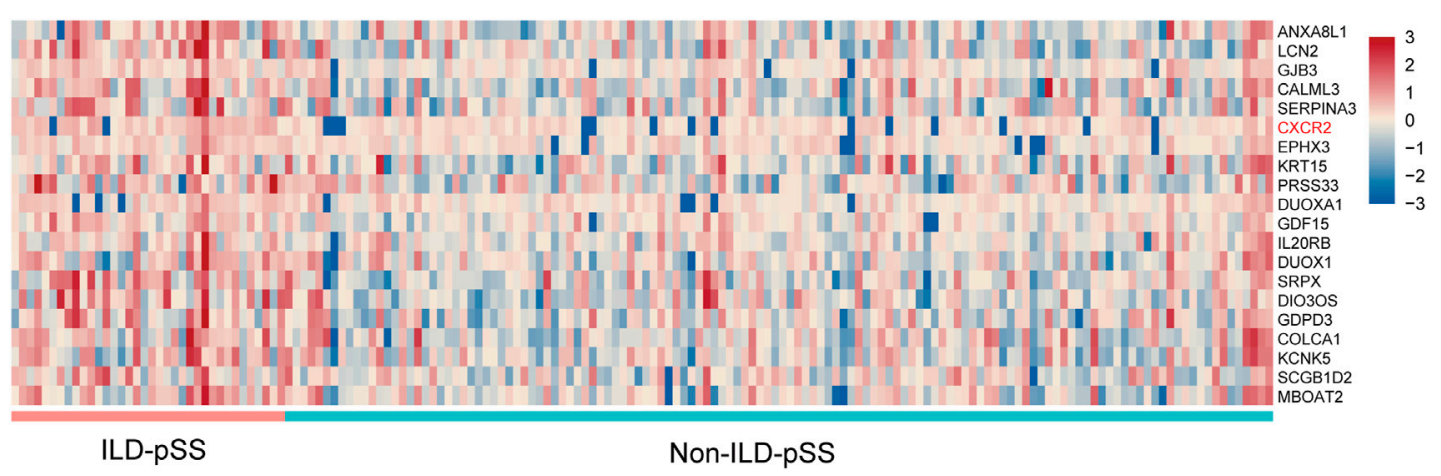

FIGURE 2 | Heatmap of the top 20 DEGs between ILD-pSS and non-ILD-pSS.

A

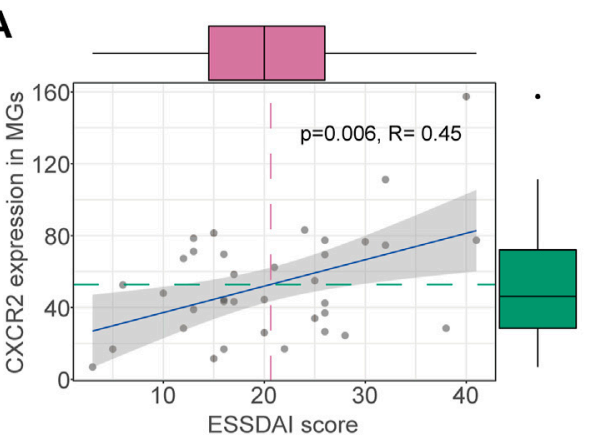

B
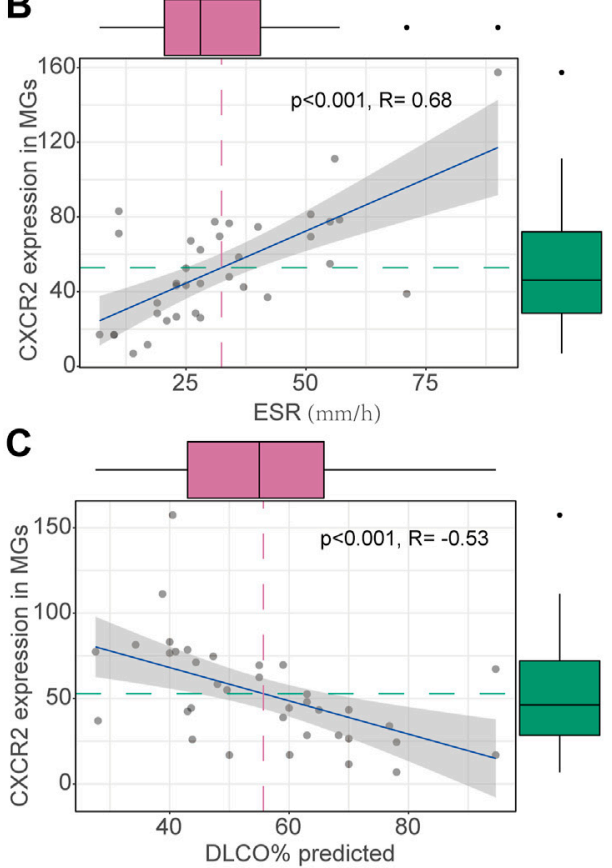

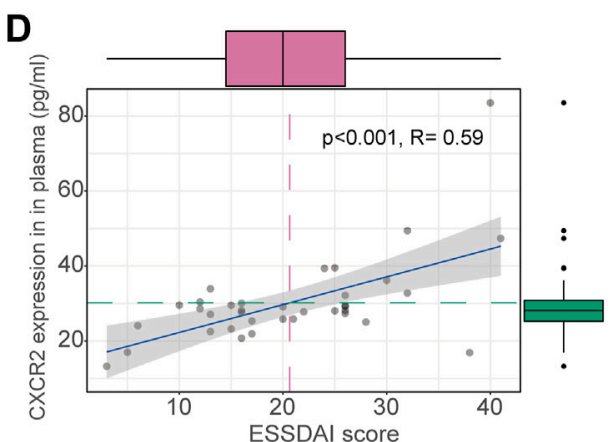

E
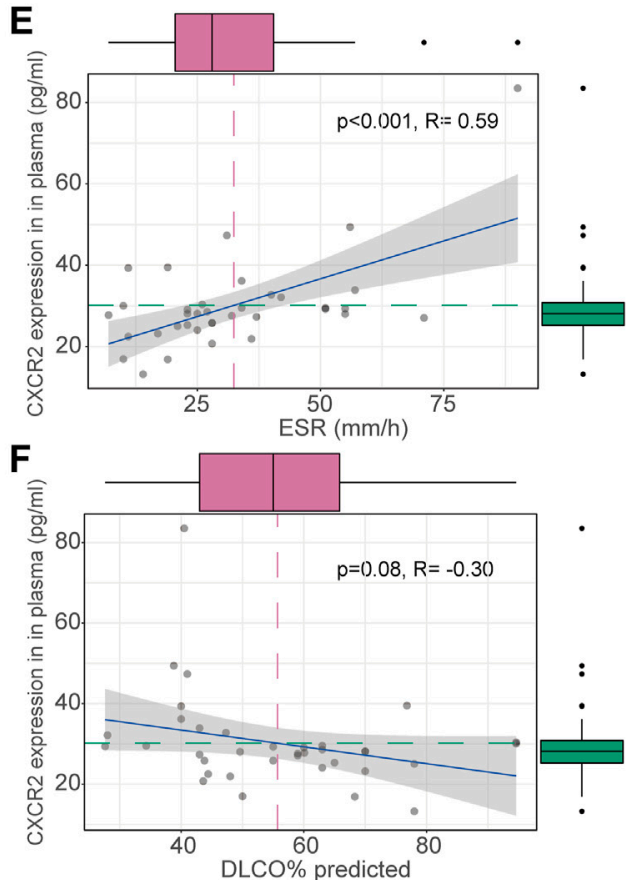

FIGURE 3 | Clinical correlations of CXCR2 expression in patients with ILD-pSS. CXCR2 gene expression in MSG was positively correlated with the ESSDAI score (A) and ESR (B), and inversely associated with DLCO (C). CXCR2 gene expression in plasma was similarly positively correlated with the ESSDAI score (D) and ESR (E), and inversely associated with DLCO (F). 


\section{Identification of DEGs in Patients with pSS-ILD}

We investigated the gene-expression profiles of MSGs from 36 patients with ILD-pSS and 128 with non-ILD-pSS using RNA sequencing. Several genes were found to be differentially expressed in ILD-pSS vs. non-ILD-pSS. The top 20 DEGs are shown in Figure 2.

\section{Clinical Correlations of Top 20 DEGs in ILD-pSS}

To identify potential biomarkers for evaluating disease severity in patients with ILD-pSS, the clinical implications of the top 20 DEGs (Figure 2) were evaluated, with clinical parameters including ESSDAI score, ESR, C-reactive protein, forced vital capacity, forced expiratory volume in $1 \mathrm{~s}$, and carbon monoxide diffusing capacity of the lung (DLCO). Among which CXCR2, a receptor for IL-8, had a higher relationship with clinical parameters than the other 19 genes (data not shown). In addition, CXCR2 was of greatest interest because of its potential role in inflammation (Figure 1A) (Russo et al., 2009; Konrad and Reutershan, 2012; Liang et al., 2016; Hoegl et al., 2017). In our study, CXCR2 was positively correlated with the ESSDAI score $(r=0.45, p=0.006$; Figure 3A) and ESR $(r=0.68$, $p<0.001$; Figure 3B). Moreover, CXCR2 gene expression inversely associated with DLCO $(r=-0.53, p<0.001$; Figure 3C). No correlations were found between CXCR2 RNA expression by RNA sequencing and the other clinical parameters investigated.

\section{Circulating Chemokine Assay of CXCR2 and IL-8}

Concentrations of CXCR2 and IL-8 in plasma were detected by ELISA in patients with ILD-pSS and non-ILD-pSS. CXCR2 levels were higher in those with ILD-pSS $(p=0.0015$; Figure 1B), but there was no significant difference in plasma IL-8 levels between the two groups $(p=0.080)$. Concentrations of CXCR2 in plasma were positively correlated with the ESSDAI score $(r=0.59, p<0.001$; Figure 3D) and ESR $(r=0.59, p<0.001$; Figure 3E) in patients with ILD-pSS. Furthermore, CXCR2 levels were inversely associated with DLCO, although this did not reach statistical significance $(r=-0.30, p=0.08$; Figure 3F).

\section{DISCUSSION}

In this study, we first compared the MSG gene-expression profiles of 36 patients with ILD-pSS and 128 patients with nonILD-pSS, and identified several genes that are up-regulated in ILD-pSS. To identify potential biomarkers for evaluating disease severity in ILD-pSS, the clinical implications of the top 20 DEGs were examined. In particular, we found that patients with ILD-pSS had abnormally increased levels of CXCR2. Moreover, the CXCR2 level correlated with clinical features in these patients, implying a potential role for CXCR2 in the progression of ILD in pSS.

CXCR2 (Rot and von Andrian, 2004) is a G-protein-coupled receptor assembled by seven transmembrane proteins that is activated by CXC chemokines containing the ELR (Glu-LeuArg) motif, including IL-8. CXCR2 is expressed by granulocytes, especially neutrophils (Kim, 2004; Bertini et al., 2012). Neutrophils are a vital component of the innate immune system and play an essential role in the progression of lung fibrosis. In addition, research has shown that CXCR2mediated neutrophil recruitment is essential for lung fibrosis (Russo et al., 2009; Konrad and Reutershan, 2012; Liang et al., 2016; Hoegl et al., 2017), and that blocking CXCR2 can reduce lung fibrosis (Russo et al., 2009). In this study, CXCR2 was found to be elevated in both the MSG and plasma of patients with ILD-pSS vs. non-ILD-pSS, with the results showing that CXCR2 may also play an essential part in organ-typical damage and circular inflammation in pSS. Although patients with ILDpSS and those with non-ILD-pSS may initially show similar clinical manifestations (Nannini et al., 2013), those with ILDpSS can have a poorer prognosis (Roca et al., 2017; Sambataro et al., 2020). Further molecular functional investigation of CXCR2 in ILD-pSS progression may have significant implications.

The ESR is a common inflammatory marker, and an elevated ESR is found associated with ILD underlying other connective tissue diseases (Young et al., 2007; Jung et al., 2018). Furthermore, previous research has demonstrated that the ESR is higher in patients with ILD-pSS vs. nonILD-pSS (Li et al., 2015; Dong et al., 2018). Our study confirmed this, with a higher ESR in patients with ILD-pSS. Moreover, CXCR2 in both MSG and plasma was highly associated with the ESR, indicating that CXCR2 may help differentiate ILD-pSS from non-ILD-pSS in some situations. The ESSDAI was developed to measure systemic disease activity in pSS (Seror et al., 2010; Risselada et al., 2012). In our study, ESSDAI scores were much higher in patients with ILD-pSS vs. non-ILD-pSS. Furthermore, ESSDAI scores were highly associated with CXCR2 levels in both MSG and plasma from patients with ILD-pSS, indicating that CXCR2 may serve as a useful candidate for evaluating disease severity. DLCO is one measure for predicting lung function. Most previous studies (Chen et al., 2016; Barnes et al., 2018) used a significant decline in DLCO to define ILD worsening. In our study, CXCR2 levels in both MSG and plasma from patients with ILD-pSS were inversely associated with DLCO, confirming the role of CXCR2 in evaluating the disease severity of ILD in those with pSS.

To our knowledge, this is the first study to suggest a possible role for CXCR2 in ILD-pSS. We observed extensive expression of CXCR2 in both MSG and plasma in patients with ILD-pSS, and many manifestations of ILD are characterized by the accumulation of inflammatory cells. CXCR2 is implicated in neutrophil infiltration and can be activated by many other CXC chemokines in inflammatory process. Therefore, the contribution of CXCR2 to the inflammatory and further fibrotic process in ILD-pSS should be further investigated. 


\section{CONCLUSION}

Our results provide new insights into the role of CXCR2 in patients with ILD-pSS. CXCR2 may serve as a potential biomarker for evaluating disease severity in this population.

\section{DATA AVAILABILITY STATEMENT}

The datasets presented in this study can be found in online repositories. The names of the repository/repositories and accession number(s) can be found below: Gene Expression Omnibus, GSE171896.

\section{ETHICS STATEMENT}

The studies involving human participants were reviewed and approved by First Affiliated Hospital of Wenzhou Medical University (approval \# 16024). The patients/participants provided their written informed consent to participate in this study.

\section{REFERENCES}

Barnes, H., Holland, A. E., Westall, G. P., Goh, N. S., and Glaspole, I. N. (2018). Cyclophosphamide for Connective Tissue Disease-Associated Interstitial Lung Disease. Cochrane Database Syst. Rev. 1, CD010908. doi:10.1002/14651858. CD010908.pub2

Bellido-Casado, J., Plaza, V., Díaz, C., Geli, C., Domínguez, J., Margarit, G., et al. (2011). Bronchial Inflammation, Respiratory Symptoms and Lung Function in Primary Sjögren's Syndrome. Archivos de Bronconeumología (English Edition) 47 (7), 330-334. doi:10.1016/j.arbres.2011.01.00310.1016/j.arbr.2011.01.006

Bertini, R., Barcelos, L., Beccari, A., Cavalieri, B., Moriconi, A., Bizzarri, C., et al. (2012). Receptor Binding Mode and Pharmacological Characterization of a Potent and Selective Dual CXCR1/CXCR2 Non-competitive Allosteric Inhibitor. Br. J. Pharmacol. 165 (2), 436-454. doi:10.1111/j.1476-5381.2011. 01566.x

Caporali, R., Bonacci, E., Epis, O., Bobbio-Pallavicini, F., Morbini, P., and Montecucco, C. (2008). Safety and Usefulness of Minor Salivary Gland Biopsy: Retrospective Analysis of 502 Procedures Performed at a Single Center. Arthritis Rheum. 59 (5), 714-720. doi:10.1002/art.23579

Chen, M. H., Chen, C. K., Chou, H. P., Chen, M. H., Tsai, C. Y., and Chang, D. M. (2016). Rituximab Therapy in Primary Sjögren's Syndrome with Interstitial Lung Disease: a Retrospective Cohort Study. Clin. Exp. Rheumatol. 34 (6), 1077-1084.

Chiu, Y.-H., Szu-Hsien Lee, T., Chao, E., Chen, I.-F., Liu, F.-C., Chiu, C.-H., et al. (2020). Application of Classification Criteria of Sjogren Syndrome in Patients with Sicca Symptoms: Real-World Experience at a Medical Center. J. Formos. Med. Assoc. 119 (1 Pt 3), 480-487. doi:10.1016/j.jfma.2019.06.012

Daniels, T. E., and Whitcher, J. P. (1994). Association of Patterns of Labial Salivary Gland Inflammation with Keratoconjunctivitis SICCA. Arthritis Rheum. 37 (6), 869-877. doi:10.1002/art.1780370615

Dong, X., Zhou, J., Guo, X., Li, Y., Xu, Y., Fu, Q., et al. (2018). A Retrospective Analysis of Distinguishing Features of Chest HRCT and Clinical Manifestation in Primary Sjögren's Syndrome-Related Interstitial Lung Disease in a Chinese Population. Clin. Rheumatol. 37 (11), 2981-2988. doi:10.1007/s10067-0184289-6

Hjelmervik, T. O. R., Petersen, K., Jonassen, I., Jonsson, R., and Bolstad, A. I. (2005). Gene Expression Profiling of Minor Salivary Glands Clearly Distinguishes Primary Sjögren's Syndrome Patients from Healthy Control Subjects. Arthritis Rheum. 52 (5), 1534-1544. doi:10.1002/art.21006

\section{AUTHOR CONTRIBUTIONS}

XfZ and SL performed most experiments. LZ, MY, XcZ and TW conducted the analysis, and prepared the figures and tables. DC, $\mathrm{CC}$, and XfZ designed the experiments and wrote the manuscript. All authors contributed to the article and approved the submitted version.

\section{FUNDING}

The current study was supported by the National Nature Science Foundation of China (81770074, 81570075, 81400035), Zhejiang Provincial Natural Science Foundation (LZ15H010001, LY18H010006), Major Program of the National Key Research and Development Program of China (2016YFC1304000), Key Laboratory of Interventional Pulmonology of Zhejiang Province (2019E10014), Zhejiang Provincial Key Research and Development Program (2020C03067), and The First Affiliated Hospital of Wenzhou Medical University (FHY2019093).

Hoegl, S., Ehrentraut, H., Brodsky, K. S., Victorino, F., Golden-Mason, L., Eltzschig H. K., et al. (2017). NK Cells Regulate CXCR2 + Neutrophil Recruitment during Acute Lung Injury. J. Leukoc. Biol. 101 (2), 471-480. doi:10.1189/jlb.3A0516227R

Jin, Y., Zhang, T., Ye, W., Zhu, X., Wang, L., and Wang, X. (2019). Clinical Profile and Associated Factors of Pulmonary Involvement in Primary Sjögren's Syndrome. Med. Clín. 153 (8), 305-311. doi:10.1016/j.medcli.2019.01.016

Jung, E., Suh, C. H., Kim, H. A., and Jung, J. Y. (2018). Clinical Characteristics of Systemic Sclerosis with Interstitial Lung Disease. Arch. Rheumatol. 33 (3), 322-327. doi:10.5606/ArchRheumatol.2018.6630

Kakugawa, T., Sakamoto, N., Ishimoto, H., Shimizu, T., Nakamura, H., Nawata, A., et al. (2018). Lymphocytic Focus Score Is Positively Related to Airway and Interstitial Lung Diseases in Primary Sjögren's Syndrome. Respir. Med. 137, 95-102. doi:10.1016/j.rmed.2018.02.023

Kelly, C., Gardiner, P., Pal, B., and Griffiths, I. (1991). Lung Function in Primary Sjogren's Syndrome: a Cross Sectional and Longitudinal Study. Thorax 46 (3), 180-183. doi:10.1136/thx.46.3.180

Kim, C. H. (2004). Chemokine-chemokine Receptor Network in Immune Cell Trafficking. Curr. Drug Targets Immune Endocr. Metabol Disord. 4 (4), 343-361. doi:10.2174/1568008043339712

Konrad, F. M., and Reutershan, J. (2012). CXCR2 in Acute Lung Injury. Mediators Inflamm. 2012, 1-8. doi:10.1155/2012/740987

Kreider, M., and Highland, K. (2014). Pulmonary Involvement in Sjögren Syndrome. Semin. Respir. Crit. Care Med. 35 (2), 255-264. doi:10.1055/s0034-1371529

Li, X., Xu, B., Ma, Y., Li, X., Cheng, Q., Wang, X., et al. (2015). Clinical and Laboratory Profiles of Primary Sjogren's Syndrome in a Chinese Population: A Retrospective Analysis of 315 Patients. Int. J. Rheum. Dis. 18 (4), 439-446. doi:10.1111/1756-185X.12583

Liang, M., Jiang, Z., Huang, Q., Liu, L., Xue, Y., Zhu, X., et al. (2016). Clinical Association of Chemokine (C-X-C Motif) Ligand 1 (CXCL1) with Interstitial Pneumonia with Autoimmune Features (IPAF). Sci. Rep. 6, 38949. doi:10.1038/ srep38949

Mavragani, C. P., and Moutsopoulos, H. M. (2014). Sjögren's Syndrome. Annu. Rev. Pathol. Mech. Dis. 9, 273-285. doi:10.1146/annurev-pathol-012513104728

Nannini, C., Jebakumar, A. J., Crowson, C. S., Ryu, J. H., and Matteson, E. L. (2013). Primary Sjögren's Syndrome 1976-2005 and Associated Interstitial Lung Disease: a Population-Based Study of Incidence and Mortality. BMJ Open 3 (11), e003569. doi:10.1136/bmjopen-2013-003569 
Natalini, J. G., Johr, C., and Kreider, M. (2019). Pulmonary Involvement in Sjögren Syndrome. Clin. Chest Med. 40 (3), 531-544. doi:10.1016/j.ccm.2019.05.002

Palm, O., Garen, T., Berge Enger, T., Jensen, J. L., Lund, M.-B., Aalokken, T. M., et al. (2013). Clinical Pulmonary Involvement in Primary Sjogren's Syndrome: Prevalence, Quality of Life and Mortality-Aa Retrospective Study Based on Registry Data. Rheumatology 52 (1), 173-179. doi:10.1093/rheumatology/ kes311

Risselada, A. P., Kruize, A. A., and Bijlsma, J. W. J. (2012). Clinical Applicability of the EULAR Sjögren's Syndrome Disease Activity Index: a Cumulative ESSDAI Score Adds in Describing Disease Severity. Ann. Rheum. Dis. 71 (4), 631. doi:10. 1136/annrheumdis-2011-200766

Roca, F., Dominique, S., Schmidt, J., Smail, A., Duhaut, P., Lévesque, H., et al. (2017). Interstitial Lung Disease in Primary Sjögren's Syndrome. Autoimmun. Rev. 16 (1), 48-54. doi:10.1016/j.autrev.2016.09.017

Rot, A., and von Andrian, U. H. (2004). Chemokines inInnate andAdaptiveHostDefense: Basic Chemokinese Grammar for Immune Cells. Annu. Rev. Immunol. 22, 891-928. doi:10.1146/annurev.immunol.22.012703. 104543

Russo, R. C., Guabiraba, R., Garcia, C. C., Barcelos, L. S., Roffê, E., Souza, A. L. S., et al. (2009). Role of the Chemokine Receptor CXCR2 in Bleomycin-Induced Pulmonary Inflammation and Fibrosis. Am. J. Respir. Cell Mol Biol 40 (4), 410-421. doi:10.1165/rcmb.2007-0364OC

Sambataro, G., Ferro, F., Orlandi, M., Sambataro, D., Torrisi, S. E., Quartuccio, L., et al. (2020). Clinical, Morphological Features and Prognostic Factors Associated with Interstitial Lung Disease in Primary Sjögren's Syndrome: A Systematic Review from the Italian Society of Rheumatology. Autoimmun. Rev. 19 (2), 102447. doi:10.1016/j.autrev.2019.102447

Seror, R., Ravaud, P., Bowman, S. J., Baron, G., Tzioufas, A., Theander, E., et al. (2010). EULAR Sjögren's Syndrome Disease Activity Index: Development of a Consensus Systemic Disease Activity Index for Primary Sjögren's Syndrome. Ann. Rheum. Dis. 69 (6), 1103-1109. doi:10.1136/ard.2009. 110619
Seror, R., Theander, E., Bootsma, H., Bowman, S. J., Tzioufas, A., Gottenberg, J.-E., et al. (2014). Outcome Measures for Primary Sjögren's Syndrome: A Comprehensive Review. J. Autoimmun. 51, 51-56. doi:10.1016/j.jaut.2013. 12.010

Shiboski, C. H., Shiboski, S. C., Seror, R., Criswell, L. A., Labetoulle, M., Lietman, T. M., et al. (2017). 2016 American College of Rheumatology/European League against Rheumatism Classification Criteria for Primary Sjögren's Syndrome. Ann. Rheum. Dis. 76 (1), 9-16. doi:10.1136/annrheumdis-2016-210571

Shiboski, S. C., Shiboski, C. H., Criswell, L. A., Baer, A. N., Challacombe, S., Lanfranchi, H., et al. (2012). American College of Rheumatology Classification Criteria for Sjögren's Syndrome: A Data-Driven, Expert Consensus Approach in the Sjögren's International Collaborative Clinical Alliance Cohort. Arthritis Care Res. 64 (4), 475-487. doi:10.1002/acr.21591

Vissink, A., and Bootsma, H. (2017). Refining the Classification Criteria for Primary Sjögren Syndrome. Nat. Rev. Rheumatol. 13 (1), 10-12. doi:10. 1038/nrrheum.2016.208

Young, A., Koduri, G., Batley, M., Kulinskaya, E., Gough, A., Norton, S., et al. (2007). Mortality in Rheumatoid Arthritis. Increased in the Early Course of Disease, in Ischaemic Heart Disease and in Pulmonary Fibrosis. Rheumatology 46 (2), 350-357. doi:10.1093/rheumatology/kel253

Conflict of Interest: The authors declare that the research was conducted in the absence of any commercial or financial relationships that could be construed as a potential conflict of interest.

Copyright (c) $2021 \mathrm{Zhu}, \mathrm{Lu}, \mathrm{Zhu}, \mathrm{Yu}, \mathrm{Wei}, \mathrm{Zhu}$, Chen and Chen. This is an openaccess article distributed under the terms of the Creative Commons Attribution License (CC BY). The use, distribution or reproduction in other forums is permitted, provided the original author(s) and the copyright owner(s) are credited and that the original publication in this journal is cited, in accordance with accepted academic practice. No use, distribution or reproduction is permitted which does not comply with these terms. 